\title{
On Applying Molecular Computation to the Data Encryption Standard
}

\author{
LEONARD M. ADLEMAN, ${ }^{1,2}$ PAUL W. K. ROTHEMUND, ${ }^{1,2}$ SAM ROWEIS, ${ }^{1,3}$ \\ and ERIK WINFREE ${ }^{1,3}$
}

\begin{abstract}
Recently, Boneh, Dunworth, and Lipton (1996) described the potential use of molecular computation in attacking the United States Data Encryption Standard (DES). Here, we provide a description of such an attack using the sticker model of molecular computation. Our analysis suggests that such an attack might be mounted on a tabletop machine using approximately a gram of DNA and might succeed even in the presence of a large number of errors.
\end{abstract}

Key words: DES, DNA computation, molecular computation, sticker model

\section{INTRODUCTION}

W ITH THEIR WORK on DES (Data Encryption Standard), Boneh et al. (1996) provided the first example of a "practical" problem which might be susceptible to molecular computation. DES is one of the most widely used cryptographic systems. It produces a 64-bit ciphertext from a 64-bit plaintext under the control of a 56-bit key. While it has been argued that special purpose electronic hardware (Wiener, 1994) or massively parallel supercomputers might be used to break DES in a reasonable amount of time, it appears that today's most powerful sequential machines would be unable to accomplish the task. We continue the work of Boneh $e t$ al. (1996) by considering the difficulty of breaking DES on the recently proposed sticker model of molecular computation (Roweis et al., 1998). While our results are encouraging, it must be stressed that the feasibility of such an attack will ultimately be decided in the laboratory.

In this paper, we consider the so called plaintext-ciphertext attack. Here the cryptanalyst obtains a plaintext and its corresponding ciphertext and wishes to determine the key used to perform the encryption. The most naive approach to this problem is to try all $2^{56}$ keys, encrypting the plaintext under each key until a key that produces the ciphertext is found. Remarkably, a significantly more efficient attack is not known and consequently this brute force approach will be the one considered here.

We begin by describing the algorithm to implement a plaintext-ciphertext attack for breaking DES at a logical level. This allows us to identify the fundamental operations we need to implement on a stickers machine, and serves as a roadmap for what follows.

\footnotetext{
${ }^{1}$ Laboratory for Molecular Science, ${ }^{2}$ Department of Computer Science, University of Southern California, Los Angeles, California.

${ }^{3}$ Computation and Neural Systems Option, California Institute of Technology, Pasadena, California.
} 


\section{THE MOLECULAR ALGORITHM}

Start with approximately $2^{56}$ identical ssDNA memory strands (Roweis et al., 1998) each 11,580 nucleotides long. We think of each memory strand as containing 579 contiguous blocks-referred to as regions in Roweis et al. (1998)- $B_{0}, B_{1}, B_{2}, \ldots, B_{578}$ each 20 nucleotides long. As is appropriate in the sticker model there are 579 stickers $S_{0}, S_{1}, \ldots, S_{578}$-one complementary to each block. (We refer to memory strands with annealed stickers as memory complexes.) Hence, we consider that each strand represents a 579-bit memory, and we sometimes use $B_{i}$ to refer to the bit which $B_{i}$ represents. Block $B_{0}$ is never set and is used later in the implementation of the algorithm (Subsection 3.1). Blocks $B_{1}$ through $B_{56}$ of the memory strands are used to store a key, the next 64 blocks, $B_{57}, \ldots, B_{120}$, will eventually encode the corresponding ciphertext, and the remainder of blocks are used for intermediate results during the computation. The stickers machine which processes the memory strands to compute the ciphertexts does so under the control of a microprocessor. Because the plaintext is the same in all cases, the microprocessor may store it; we do not need to represent the plaintext on the memory strands. Now, given a plaintext-ciphertext pair, the algorithm is performed in three steps:

1. Input step: Initialize the memory strands to form memory complexes representing all $2^{56}$ keys.

2. Encryption step: On each memory complex compute the ciphertext corresponding to the encryption of the plaintext under that complex's key.

3. Output step: Select the memory complex whose ciphertext matches the given ciphertext, and read the corresponding key.

The bulk of the work is performed during the second step, where DES data encryption occurs, so we outline it below. Our interest is in demonstrating how DES can be implemented on a molecular computer, and for these purposes the exact details of DES are unnecessary. For details, see (National Bureau of Standards, 1977). We will instead focus on the essential operations required in DES, and how these operations are combined to effect the full algorithm.

DES is a 16-round cipher. In each round a new 32 bit intermediate result is produced. These are designated $R_{1}, \ldots, R_{16}$. We store $R_{15}$ and $R_{16}$ in locations $B_{57}$ through $B_{120}$ (adjacent to the key), while $R_{1}, \ldots, R_{14}$ are stored in locations $B_{121}$ through $B_{568}$. Essentially, $R_{15}$ and $R_{16}$, taken together, form the desired ciphertext. (We encode the ciphertext adjacent to the key for implementation reasons explained in Subsection 3.4.) The left 32-bits and right 32-bits of the plaintext are referred to as $R_{-1}$ and $R_{0}$, and are known to the controlling microprocessor.

Bits $B_{569}$ through $B_{578}$ are used as a workspace and are written and erased during the course of the computation. Hence, unlike the other bits that are used in a "write once" fashion, these bits may be cleared; for implementation reasons, we always clear the entire workspace at once.

Essentially, $R_{i}$ is obtained from $R_{i-1}$ and $R_{i-2}$ by the following computation:

$$
R_{i}=R_{i-2} \oplus S\left(E\left(R_{i-1}\right) \oplus K_{i}\right)
$$

where $\oplus$ denotes exclusive or (x-or), $K_{i}$ denotes a round dependent selection of 48 bits from the key, $E$ denotes the expand function which takes the 32 bits of $R_{i-1}$ and repeats or permutes them to yield 48 bits, and $S$ denotes the $S$-function which takes a 48-bit input and maps it to a 32-bit output. The function $E$, the function $S$ and the selection $K_{i}$ are hard-coded, like the plaintext, into the microprocessor.

In fact, the S-function can be separated into eight independent 6-bit to 4-bit functions known as S-boxes. Hence, each $R_{i}$ may be computed in eight independent operations each of which produces a 4 bit chunk of the result. A given chunk is a function of 16 input bits: 6 bits of $R_{i-1}, 6$ bits of $K_{i}$ and 4 bits of $R_{i-2}$. We describe the computation of a chunk below:

1. Six bits of $R_{i-1}$ and 6 bits of $K_{i}$ are x-ored to produce a 6-bit result which is then stored in the workspace locations $B_{569}, \ldots, B_{574}$.

2. One of the S-box functions is applied to bits $B_{569}, \ldots, B_{574}$ and the 4-bit result is stored in the workspace locations $B_{575}, \ldots, B_{578}$.

3. Bits $B_{575}, \ldots, B_{578}$ are x-ored with 4 bits of $R_{i-2}$ to produce the desired chunk of $R_{i}$ which is then stored in the appropriate four blocks of the intermediate result bits $B_{57}, \ldots, B_{568}$.

4. If the chunk being computed is not the last chunk of $R_{16}$, the entire workspace, bits $B_{569}, \ldots, B_{578}$, is cleared in preparation for future use. 
The positions on each memory complex of the 16 input bits required to compute a given chunk depend only on the chunk number $(1, \ldots, 8)$ and the round number $(1, \ldots, 16)$, though the $0 / 1$ value of those bits will vary from memory complex to memory complex. The controlling microprocessor knows which positions contain these bits (they are hard-coded) and knows the X-or or S-box which it needs to apply.

We see, then, that encrypting a plaintext with DES comes down to a process of either (1) selecting 2 bits, producing their $\mathrm{x}$-or, and writing the result in a new bit, or (2) selecting 6 bits, applying an S-box, and writing the resulting 4 bits.

\section{IMPLEMENTATION}

We now turn to implementing the algorithm on a stickers machine. Such a machine, as described in (Roweis et al., 1998), may be thought of as a "parallel robotic workstation." It consists of a rack of tubes (data tubes, sticker tubes, and operator tubes), some robotics (arms, pumps, heater/coolers, connectors, etc.) and a microprocessor that controls the robotics. Roweis et al. (1998) assume that the components of the robotics and a set of three data or operator tubes may be arranged to perform any of the following four operations: separate, combine, set and clear.

We assume that the robotics are capable of an extended set of operations:

1. Parallel separate. The robotics can separate the DNA from each of 32 data tubes into two more data tubes by using 32 separation operator tubes at once.

2. Parallel combine. The robotics can combine the DNA from 64 different data tubes into one data tube at once. We assume that the blank operator tube used for a combine in (Roweis et al., 1998) is really just a connector which is part of the robotics.

3. Parallel set. The robotics can, using one sticker tube with stickers $S_{k}$, set the bit $B_{k}$ on the complexes in 64 different data tubes as once. We assume the sticker operator tube used for set in (Roweis et al., 1998) is just a filter that can be built into the robotics.

4. Clear. The robotics can clear the workspace bits on all complexes in one data tube. We assume that the stickers on the workspace are removed simultaneously. Hence the workspace blocks may be implemented using so called weak regions (Roweis et al., 1998). Again, we assume the sticker operator tube used for clear in (Roweis et al., 1998) is just a filter that can be built into the robotics.

Hence, we perform the above four operations using just data tubes that may hold DNA memory complexes, sticker tubes that are (for the purpose of the computation) an inexhaustible source of a particular sticker $S_{k}$, and separation operator tubes that hold probes for a particular block $B_{k}$.

In the following subsections we describe, where applicable, the implementation of the molecular algorithm using these operations and, for the purposes of estimating the time and space required by a stickers machine we keep track of the following three resource quantities:

1. Total steps. We define the number of steps as the number of parallel separations, parallel combines, parallel sets or clears that any given complex experiences after it has been initialized. Hence, we count the operation of the robotics on a large number of tubes in parallel as a single step and ignore the (perhaps serial) process of moving data and operator tubes.

2. Total rack tubes. We define the number of rack tubes to be the total number of data tubes, sticker tubes, and separation operator tubes used during the computation. All of the tubes are reusable, so we only need copies of a tube if a particular kind of tube must be used more than once in a parallel operation. We note that we never need duplicates of sticker tubes-our robotics are incapable of using more than one sticker tube at once. We will however need duplicate separation operator tubes and many data tubes since we often wish to separate complexes in several different data tubes on the same bit $B_{i}$ at once.

3. Maximum number of active tubes per operation. We define the number of active tubes, for any time during the computation, as the number of tubes which the robotics have removed from the rack and are currently processing. Note that the maximum number of active tubes defines the width of the parallelism used by our algorithm and hence it must match the parallelism built into our robotics.

We note that these quantities are applicable for only for the computation of the ciphertexts (step 2 of the molecular algorithm) and the selection of the given ciphertext (the first part of step 3 of the molecular 
ADLEMAN ET AL.

algorithm). These parts of the molecular algorithm require only the operations given above and hence are performed using the stickers machine. The initialization of the memory complexes and final reading of the key, however, use some operations that are not included above (i.e., dividing the contents of a tube into two tubes, PCR amplification, ligation, etc.) These special operations are used at most once during the implementation of the molecular algorithm and we assume that they could be performed by the human operator of the stickers machine in a reasonably short amount of time (a few hours) and in a small space (a few tubes). Hence, they are ignored in the final discussion of the resources required by a stickers machine (Subsection 3.5).

\subsection{Initialization of the memory strands}

First we must create the initial tube encoding keys. Our desire is to have each of the $2^{56}$ memory strands store a different key. This might be accomplished, for example, in the following way:

1. Divide the memory strands into two tubes, $A$ and $B$.

2. Add an excess of $S_{1}$ through $S_{56}$ to tube $A$ and allow them to saturate the first 56 blocks on each strand.

3. Use the complement of $B_{0}$ as a probe to separate the memory complexes in tube $A$ from the excess stickers.

4. Add tube $B$ to tube $A$.

5. Heat and cool tube $A$ to randomly reanneal the stickers.

The memory complexes produced by this process appear to be reasonably modeled with a Poisson distribution; it is expected that approximately $63 \%$ of keys will be represented and that, on average, there is one of each key. Hence, if no errors are committed during the computation, we have a reasonable chance of recovering the key for a ciphertext of interest. Of course, the chance of succeeding can be increased by starting with more memory strands. To insure that approximately $95 \%$ of the keys are represented and that on average three copies of a key are present, we could simply use three times as much DNA. These issues are discussed in more detail in the Section 4.

\subsection{Implementing the fundamental operations}

As discussed in Section 2, the DES encryption algorithm is a composition of just two simple functions:

- $\mathrm{x}$-ors which are 2-bit input to 1-bit output maps,

- S-boxes which are 6-bit input to 4-bit output maps.

The computation of the 2-bit to 1-bit x-or function is illustrated in Figure 1.

We now describe the computation of the $\mathrm{x}$-or function $B_{k}=B_{i} \oplus B_{j}$ in greater detail, give the overhead required, and generalize this to an $n$-bit to $m$-bit function:

A. Parallel separate the sample two times to yield four data tubes, one for each possible value $B_{i} B_{j}$. This is accomplished by first using one separation operator tube specific for $B_{i}$ and then, in parallel, using two separation operator tubes specific for $B_{j}$. (In Figure 1, the bits being considered are shaded gray.) Roweis

- et al. (1998) model a single separation as an operation involving three active tubes at once: a source data tube, a separation operator tube, and one of two destination data tubes picked up and filled by the robotics in sequence. Hence, during each single separation, three tubes are active at once and three data tubes are used. During the second parallel separation above, then, six data tubes are used and six tubes are active.

For an $n$-bit to $m$-bit function this generalizes to:

Parallel separate the sample $n$ times to yield $2^{n}$ data tubes, one for each value of the $n$-bit input. This requires $2^{i-1}$ separation operator tubes for the ith parallel separation (a total of $2^{n}-1$ ). Hence, for the $n$th parallel separation, $3 \times 2^{n-1}$ data tubes are required and $3 \times 2^{n-1}$ tubes are active.

B. Parallel set $B_{k}$ to 1 with an $S_{k}$ sticker for all tubes for which this is applicable. For an x-or this is only applicable when $B_{i} B_{j}=01$ or 10 but for a general 2-1 function this may require the addition of a sticker to any subset of the four data tubes in parallel. This requires one sticker tube and four data tubes, a total of five active tubes.

For an $n$-bit to $m$-bit function this generalizes to:

Parallel set a (possibly different) subset of the $2^{n}$ data tubes $m$ times using a total of $m$ sticker tubes. This requires $2^{n}+1$ active tubes. Note that the subset of data tubes to which the parallel set is applied is determined solely by the algorithm stored in the microprocessor. 


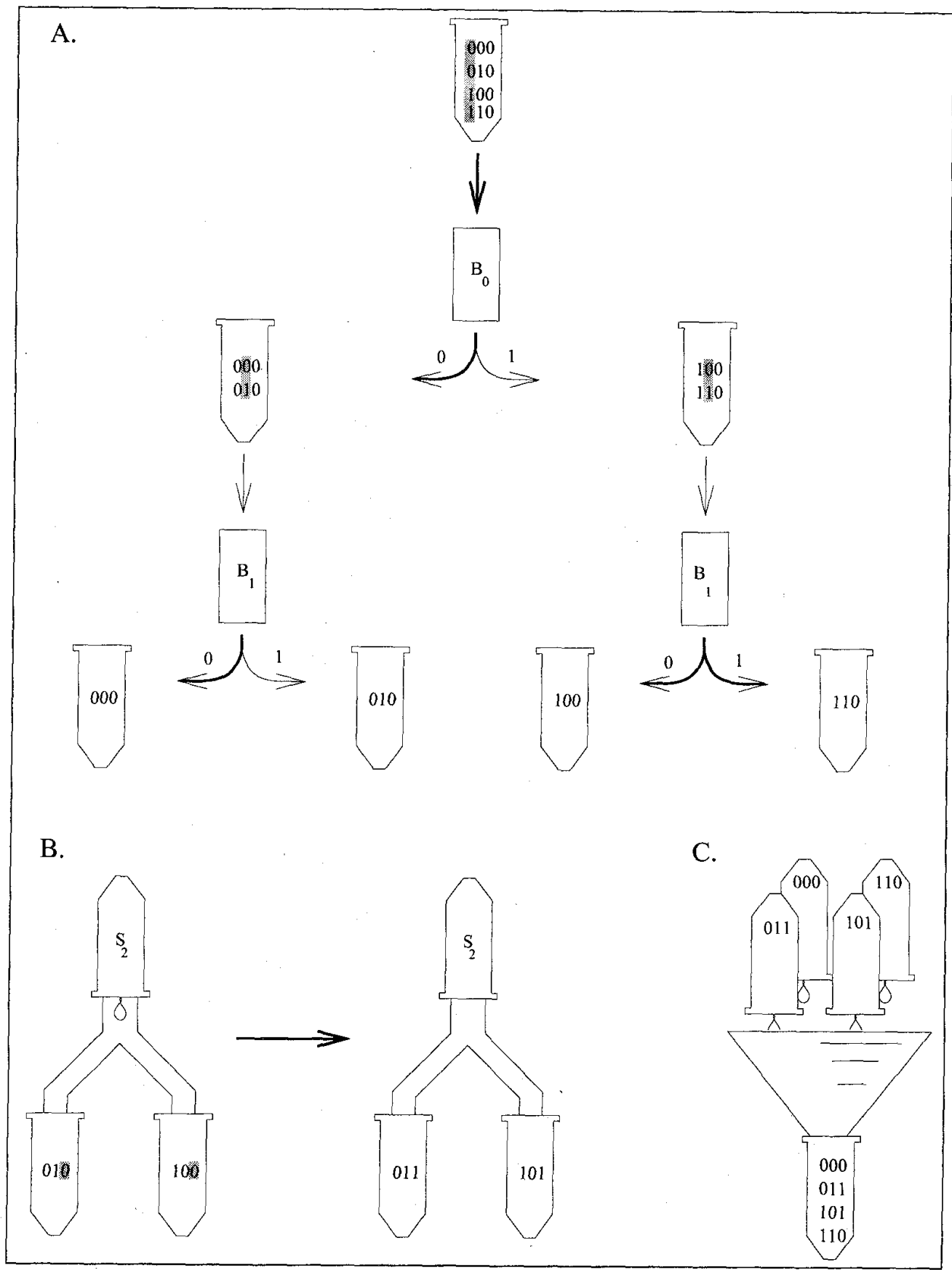

FIG. 1. Computation of the 2-bit to 1-bit x-or function: $B_{2}=B_{0} \oplus B_{1}$.

C. Parallel combine the contents of all four data tubes into one data tube. This requires five data tubes and hence five active tubes.

For an $n$-bit to $m$-bit function this generalizes to:

Parallel combine the contents of all $2^{n}$ data tubes into one data tube. This requires $2^{n}+1$ data tubes and $2^{n}+1$ active tubes.

At the end of our x-or operation, all of our DNA has been returned to a single tube.

In general, an $n$-bit to $m$-bit function requires $n+m+1$ steps, $2^{n}-1$ separation operator tubes (specific to various bits), $m$ sticker tubes, a maximum of $3 \times 2^{n-1}$ data tubes, and a maximum of $3 \times 2^{n-1}$ active tubes.

At this point we can see that one of the resource quantities of interest, the maximum number of active tubes has already been specified. A 6-bit to 4-bit S-box is the biggest $n$-bit to $m$-bit function we implement in DES 
so we never use more than 96 active tubes. (The only other operation used in the algorithm, clear, uses but one active tube.)

The number of rack tubes is the sum of the data, sticker and separation tubes used but we may not simply sum up these numbers for an S-box and regard the result as its contribution to the computation. Data tubes are interchangeable so we know that the maximum number used in an S-box-96-will be the data tube contribution to the total rack tube count. However, sticker tubes and separator tubes have an identity, they are associated with a particular block of the memory complexes. For the calculation of rack tubes (and total steps as well), we need to consider more details of the molecular algorithm.

Thus, in the next subsection, we consider how the x-ors and S-boxes used to compute DES are composed, count the total number of steps, and discuss ways to plan our use of separation operator tubes and sticker tubes to minimize the total number of rack tubes.

\subsection{Computing the ciphertexts}

We recall that the basic unit of computation in our molecular algorithm is a 4 bit chunk that is computed using a composition of $\mathrm{x}$-ors and S-boxes which takes 16 bits of input. For any given chunk the positions of these 16 input bits are the same for every memory complex. Thus the positions of the input bits are hard-coded into the microprocessor and we ignore their exact values in what follows.

From our consideration of $n$-bit to $m$-bit functions, we know that four steps are required to compute an $\mathrm{x}$-or and 11 steps are required to compute an S-box. From this it follows that to compute a chunk requires $6 \times 4+11+4 \times 4=51$ steps. After a chunk is computed, if the workspace is to be used again, it must be cleared. To complete the computation requires computing $16 * 8=128$ chunks, and clearing the workspace 127 times; hence, the total number of steps required is 6,655 .

During the computation of an $\mathrm{x}$-or, one separation operator tube is required to separate on the first bit, and two separation operator tubes are required to separate (in parallel) on the second bit. To economize on separation operator tubes when performing $\mathrm{x}$-ors, we choose to separate first on a bit for which there are many instances of that "kind" of bit on the memory complex, and second on a bit for which there are only a few instances of that "kind" of bit. Hence, for each x-or involving a bit of $R_{i-2}$ (of which there are 448 possibilities from $R_{1}, \ldots, R_{14}$ ) and a bit from the workspace $B_{575}, \ldots, B_{578}$ (of which there are only four), we separate on the $R_{i-2}$ bit first and the workspace bit second. Likewise, for each x-or involving a bit of $R_{i-1}$ (of which there are 480 possibilities from $R_{1}, \ldots, R_{15}$ ) and $K_{i}$ (of which there are 56 possibilities), we separate on the $R_{i-1}$ bit first and the key bit second. It follows that for each of the bits form $R_{1}, \ldots, R_{15}$ one separation operator tube specific to that bit is needed and, for each of the bits of $K$ and each of the bits $B_{575}, \ldots, B_{578}$ two separation operator tubes specific to that bit are needed. Hence, these bits require a total of $480+2 \times 56+2 \times 4=600$ separation operator tubes.

The implementation of the S-boxes demonstrates another way that we may economize on separation operator tubes: frequently used subcomputations do not require that we use up a new bit (and consequently another separation operator tube) every time they are run. Instead their input and output may be stored on a rewritable region of the memory complex-hence, our placement of the input to the S-boxes in the first six workspace positions and the output in the last four workspace positions. Because of this, even though there are eight different S-boxes (one for each chunk), they all may use the same separation operator tubes and sticker tubes (though, under control of the microprocessor, the stickers are applied differently for each S-box). The S-boxes, then, only require an additional 63 separation operator tubes to separate the DNA into all possible 6-bit strings $B_{569}, \ldots, B_{574}$.

Thus, to complete the ciphertext generation stage of the algorithm, we require 663 separation operator tubes. In all, $512+10=522$ sticker tubes are required to hold the stickers used to write the intermediate results and workspace bits. (Recall that our robotics only use one sticker tube at a time.) The greatest number of data tubes used during the computation is 96, during the final parallel separation step of an S-box computation. In total, $663+522+96=1,281$ rack tubes are required to compute the ciphertexts.

\subsection{Selecting the given ciphertext and reading the correct key}

Once the ciphertexts have been computed, the desired key can be selected by searching for the complex which has the given ciphertext encoded next to its key. This requires 64 separation steps. Upon isolating the desired complex, it is necessary to read its key. Reading could be attempted using single molecule detection 
and a binary tree decoding as described in (Roweis et al., 1999). However, it is not clear that such an approach can be satisfactorily carried out in lab. Below we describe two additional approaches, each of which entails some modification of the methods described in the previous sections:

- In the first approach, the memory strands are $5^{\prime}$ biotinylated. Once the ciphertexts have been computed, as described in the previous sections, the memory complexes are transformed en masse into single-stranded form. One way to accomplish this is as follows:

1. For each sticker $S_{0}, \ldots, S_{120}$ create a new 0 -sticker, $S_{i}^{\prime}$, which shares the $3^{\prime}$ and $5^{\prime} 8$-mers with $S_{i}$ but which differs from it in the middle 4-mer.

2. Add an excess of the $S_{i}^{\prime}$ to the final solution under conditions which favor annealing despite the mismatches.

3. Add ligase. Each memory complex now has a heteroduplex region in its key-ciphertext section where the nonmemory strand consists of a sequence of (regular) stickers and 0 -stickers. Note that our original decision to place the ciphertext next to the key on the memory strands is no longer a mystery. Our placement minimizes the number of ligations which must be successful for a strand which encodes both the ciphertext and key to form.

4. Separate out the biotinylated memory strands using a streptavidin-coated solid support and retain the new strands as the library of solutions.

This process essentially converts Sticker memory complexes into Lipton style memory strands (Lipton, 1995) for which each block has one of two unqiue sequences, one for 0 and another for 1 . A ciphertext of interest may be selected (by applying the usual 64 separation steps), PCR amplified (using $S_{0}^{\prime}$ and the complement of the last ciphertext bit as primers), and read (with standard DNA sequencing).

Notice that, once created, the library of solutions can be replicated by PCR and hence multiple copies can be made. Each such copy is essentially a codebook consisting of (key, ciphertext) pairs. This codebook has approximately $2^{56} \times(56$ key bits +64 ciphertext bits $)=2^{63}$ bits of information (the equivalent of approximately 1 billion 1 gigabyte CDs [compact discs]) but occupies a dry volume of approximately one-seventh of a teaspoon. As Boneh et al. (1995) and Lipton (1995) have noted, such a codebook could be widely distributed and used to speed up subsequent attacks on DES.

- In the second approach to reading, instead of converting to Lipton style memory strands after the computation of ciphertexts, a Lipton-Sticker hybrid model for encoding the initial memory complexes is used.

1. Make single-stranded DNAs representing all $2^{56}$ keys using the encoding of Lipton. Additionally, guarantee that each key strand begins and ends with the same short PCR primer.

2. Ligate, to each of the Lipton key strands, identical 522-bit Sticker memory strands.

3. Proceed to compute DES as usual.

4. Perform 64 separations on the result to obtain the complex carrying the desired ciphertext.

5. Perform PCR using the primers that bound the Lipton style key sequence. The key sequence will be amplified exponentially and may be sequenced.

We note that all of the techniques for selecting a desired ciphertext discussed here and in Roweis et al. (1999) would require 64 separation operations so we approximate that this process requires an additional 64 steps. Further, we note that to effect all 64 separations an additional 32 separation operator tubes specific to the bits of $R_{16}$ (in addition to the 32 separation operator tubes already counted for $R_{15}$ ) are required.

\subsection{Discussion}

In summary, to find the key for a DES-encoded plaintext-ciphertext pair, we first create memory complexes representing all keys, then we compute the ciphertext corresponding to each key $(6,655$ steps), and finally we select and read the key of interest (64 steps). This requires a total of 6,719 steps, each of which is one of the operations described above.

The actual running time for the algorithm depends on how fast the operations can be performed. If we assume, as we might if a graduate student had to perform each operation, that each operation requires 1 day, then the computation will require 18 years. If each operation requires $1 \mathrm{~h}$ (Boneh et al. [1996] assume $2.4 \mathrm{~h}$ ), 
then the computation will require approximately 9 months. If each operation can be completed in 1 min, perhaps using a robotic stickers machine, then the computation will take 5 days. Finally, if the effective duration of a step can be reduced to $1 \mathrm{sec}$, perhaps by running the algorithm in a continuous flow parallel refinery (Roweis et al., 1999), then the effort will require $2 \mathrm{~h}$.

The size of the rack is dictated by the amount of DNA used. When the $2^{56}$ memory complexes have half of their sticker positions occupied, as we expect will be the case at the end of the computation, they weigh approximately $0.7 \mathrm{~g}$ and, in solution at $5 \mathrm{~g} / \mathrm{L}$, would occupy approximately $140 \mathrm{ml}$. Hence, the volume of the 1,313 rack tubes ( 1,281 for ciphertext computation; an additional 32 for ciphertext selection) need be no more than $140 \mathrm{ml}$ each. It follows that the rack tubes occupy, at most, $184 \mathrm{~L}$ and can, for example, be arrayed in a rack $1 \mathrm{~m}$ (approximately 39 inches) long and wide and $18 \mathrm{~cm}$ (approximately 7 inches) deep. Since the robotics must be able to operate on 96 active tubes in parallel we approximate the volume required by the robotics, give or take some arms and pumps, as $13 \mathrm{~L}$-one-fourteenth the volume of the rack. The microprocessor is likely to be quite small. Thus, it is reasonable to assume the entire machine would fit on a desktop.

It is worth pointing out that, for much of the computation, the robotics are not processing at full capacity ( 96 tubes) and many of the tubes are sitting idle. Hence, it may be possible to increase the parallelism significantly by pipelining the computation.

\section{ANALYSIS OF ERRORS}

We say that an error has occurred whenever a memory complex is transformed in an unintended way or ends up in an unintended place. Hence, there are many kinds of errors that can occur during the operation of our DNA computer: strands can break, stickers can fall off one memory complex and reanneal to another, complexes can be "lost" on the walls of a tube, complexes can end up in the wrong tube following a separation, etc.

For each operation, we define its error rate to be the fraction of molecules that commit an error during that operation. Some operations are more prone to errors than others. To simplify our analysis, we define $E$ to be the error rate of the worst operation and assume that all of the operations have error rate $E$. Note that $1-E$ corresponds to what chemists call yield. Hence an error rate of $10^{-4}$ corresponds to a step yield of $99.99 \%$.

Given an input ciphertext-plaintext pair, it is possible that several different keys map the ciphertext into the plaintext (though for DES, it seems unlikely that the number of such keys would be large). All such keys will be called winning keys. Under ideal conditions, after the codebook is created and the separation on the input ciphertext performed, we are left with a final tube with the following properties:

1. For each winning key, there is at least one complex encoding it.

2. All complexes that are there encode winning keys.

In reality, this can fail for either of two reasons. First, complexes encoding winning keys may be missing, either because they were not created during initialization or because they encountered an error during the computation. Second, there may be distractors: complexes which do not encode winning keys, but due to errors end up in the final tube anyway. In Subsection 4.1, we analyze the probability that a winning key has a complex encoding it in the final tube. In Subsection 4.2, we calculate the expected number of distractors in the final tube.

We make the following assumptions:

1. After the initialization step, each complex encodes a 56-bit key chosen at random (i.e., chosen from the space of all 56-bit keys with equal probability).

2. DES with the input plaintext maps each of the keys to a random ciphertext.

3. A complex that encounters an error during the computation produces a random ciphertext (i.e., unrelated to the ciphertext normally associated with that complex's key).

\subsection{Probability that a winning key has a complex encoding it in the final tube}

In the computation above, we began with $2^{56}$ memory strands. It will be convenient to carry out the analysis in greater generality. We now assume that we begin with $2^{56} X$ memory strands, where $X$ is a positive rational. Informally, $X$ is the factor by which we multiply our original amount of DNA.

Let $K_{w}$ be a winning key. Following initialization, the number of memory complexes which encode $K_{w}$ is given by a binomially distributed random variable $(n, p)=\left(2^{56} X, \frac{1}{2^{56}}\right)$. 
The probability that a memory complex makes it correctly through all 6,655 steps of the computation and 64 steps of the selection process (in total 6,719 steps) is given by:

$$
S=(1-E)^{6,719}
$$

Hence, after the computation, the number of memory complexes in the final tube which encode $K_{w}$ is given by a binomially distributed random variable $(n, p)=\left(2^{56} X, S \frac{1}{2^{56}}\right)$. Because $2^{56} X$ is very large and $S \frac{1}{2^{56}}$ is very small, this distribution may be approximated by a Poisson distribution with Poisson parameter $\lambda=n p=S X$. From this it follows that the expected number of complexes encoding $K_{w}$ in the final tube is $S X$ and that the probability that a complex encoding $K_{w}$ is in the final tube is:

$$
\left(1-e^{-S X}\right)
$$

In particular, when $X=1 / S$, the expected number of complexes encoding $K_{w}$ in the final tube is one and the probability that a complex encoding $K_{w}$ is in the final tube is $63 \%$. We will refer to $63 \%$ as a reasonable chance.

\subsection{Number of distractors in the final tube}

For a memory complex $M$, let $H(M)$ denote the Hamming distance of the ciphertext encoded on $M$ from the input ciphertext. For $M$ to enter the final tube, $H(M)$ errors must occur during the final 64 separation steps. By our assumptions, after the computation of the codebook, each memory complex (whether it has encountered an error or not) encodes a ciphertext which is a random 64 bit string. It follows that the Hamming distances associated with memory complexes will be a binomially distributed random variable $(n, p)=(64,0.5)$. Hence, the probability that $H(M)=L$ is

$$
\left(\begin{array}{c}
64 \\
L
\end{array}\right)\left(\frac{1}{2}\right)^{64-L}\left(\frac{1}{2}\right)^{L}=\left(\begin{array}{c}
64 \\
L
\end{array}\right) \frac{1}{2^{64}}
$$

The probability of complex $M$ with Hamming distance $H(M)=L$ making it through the 64 step selection process is given by the probability that it correctly negotiates $64-L$ separations for which it matches the input ciphertext times the probability that it commits an error at the $L$ separations for which it mismatches the correct ciphertext:

$$
(1-E)^{64-L} E^{L}
$$

There are $2^{56} X$ complexes in the codebook so the expected number of distractors is given by:

$$
\sum_{L=0}^{64} \frac{2^{56} X}{2^{64}}\left(\begin{array}{c}
64 \\
L
\end{array}\right)(1-E)^{64-L} E^{L}=\frac{X}{256}
$$

Perhaps surprisingly, the expected number of distractor molecules is independent of the error rate. Note, in particular, that for $X=1$ the expected number of distractors is less than one.

\subsection{Feasibility}

Combining the results from 4.1 and 4.2 gives Table 1, which, shows for various error rates, the amount of DNA that must be pushed through the DES computation to insure that there is a reasonable chance $(63 \%)$ of getting a winning key in the final tube. Table 1 also records the expected number of distractors which will be present in the final tube.

TABLE 1. QUANTITY OF DNA REQUIRED AND NUMBER OF DISTRACTORS AS A FUNCTION OF ERROR RATE

\begin{tabular}{lcccc}
\hline & \multicolumn{4}{c}{ Achievable error rate $E$} \\
\cline { 2 - 5 } & 0 & $10^{-4}$ & $10^{-3}$ & $10^{-2}$ \\
\hline$X$ & 1 & 2 & 830 & $2.1 \times 10^{29}$ \\
Grams of DNA required & 0.7 & 1.4 & 580 & $1.5 \times 10^{29}$ \\
Distractors & 0.004 & 0.008 & 3.2 & $8.3 \times 10^{26}$ \\
\hline
\end{tabular}


This indicates that for an error rate of $10^{-4}$, a little more than $1 \mathrm{~g}$ of DNA is needed and the final tube will usually have no distractors. If an error rate of $10^{-3}$ is achievable, then less than a kilogram of DNA is needed and only a small number of distractors need be dealt with before finding the correct answer. However, if an error rate of $10^{-2}$ is the best that is attainable, then huge amounts of DNA are needed (approximately 23 Earth masses) to have a reasonable chance that a winning key ends in the final tube, but even then it will have to be distinguished from colossal number of distractors - clearly an unacceptable situation. In such cases, where only very high error rates are possible, techniques like those described in (Roweis et al., 1999; for example, a refinery algorithm) may be used to reduce the amount of DNA required. Section 9 of Roweis et al. (1999) gives a brief analysis of the application of a refinery algorithm to DES.

\section{CONCLUSIONS}

We wish to emphasize that our description of an attack on DES is, at this point, entirely theoretical and whether it can be carried out in the lab remains to be seen. Huge challenges remain. For example, as yet, we have been unable to perform separations (or any of the sticker operations) in the lab with error rates approaching $10^{-4}$. Nonetheless, the analysis presented in this paper demonstrates (at least in principle) two things:

- "Real problems" can be solved with small machines that do not require huge amounts of DNA and use little or no enzymes.

- Error rates similar to those normally demanded of electronic-computers are not required.

If the attack on DES described here can be carried out in the lab, then some other cryptosystems might also be vulnerable to this approach. Indeed, the small size of the machine we describe suggests that systems like the 64-bit key FEAL (fast data encipherment algorithm) cryptographic system of Shimizu-Miyaguchi (Shimizu and Miyaguchi, 1988) might be susceptible to such an attack.

Finally, there are several messages for cryptography in these findings. It seems appropriate to reconsider one of the "axioms" of cryptography: Improvements in computational power always favor the cryptographer over the cryptanalyst. This is almost certainly untrue. The analysis presented here suggests the possibility of computers with superparallelism that can help the cryptanalyst immensely, yet provide no help for the cryptographer. Even if DNA computers prove infeasible, it is possible that new machines capable of superparallelism may make cryptosystems like DES insecure.

The DNA computer is an example of a super-parallel machine with very slow processors (complexes of DNA). The potential vulnerability of DES arises for two reasons. First, the key space is insufficiently large. Second, the DES algorithm is "too short." The fact that only 6,655 steps are needed to do an encryption allows the slow DNA processors to finish their encryptions in (at least in theory) a reasonable amount of time. Hence, the much valued throughput speed of DES and similar systems may carry with it a potential vulnerability to superparallel machines with slow processors.

\section{ACKNOWLEDGMENTS}

L.M.A. and P.W.K.R. are supported in part by the National Science Foundation under grant CCR-9403662 and the Sloan Foundation. S.R. is supported in part by the Center for Neuromorphic Systems Engineering as a part of the National Science Foundation Engineering Research Center Program under grant EEC-9402726 and by the Natural Sciences and Engineering Research Council of Canada. E.W. is supported in part by National Institute for Mental Health (NIMH) training grant no. 5 T32 MH 19138-06 and also by General Motors' Technology Research Partnerships program.

\section{REFERENCES}

Boneh, D., and Lipton, R. 1995. Batching DNA computations. CS-TR-489-95. Princeton University.

Boneh, D., Dunworth, C., and Lipton, R. 1996. Breaking DES using a molecular computer, 37-65. In Lipton, R.J. and Baum, E.B., eds, DNA Computers : Proceedings of a DIMACS Workshop, DIMACS Series in Discrete Mathematics and Theoretical Computer Science, American Mathematical Society, Providence, RI. 
Lipton, R. 1995. Using DNA to solve NP-complete problems. Science 268, 542-545.

National Bureau of Standards. 1977. Data encryption standard. FIPS pub. no. 46. U.S. Department of Commerce.

Roweis, S., Winfree, E., Burgoyne, R., et al. 1998. A sticker-based model for DNA computation. J. Comput. Biol. 5, $615-629$.

Roweis, S., and Winfree, E. 1999. On the reduction of errors in DNA Computation. J. Comput. Biol. 6, 65-75.

Shimizu, A., and Miyaguchi, S. 1988. Fast data encipherment algorithm FEAL, 267-278. In Chaum, D., and Price, W.L., eds. Lecture Notes in Computer Science 304, Springer-Verlag, New York.

Wiener, M. 1994. Efficient DES key search. TR-244. School of Computer Science, Carleton University.

Address reprint requests to:

Paul W. K. Rothemund

Department of Computer Science

University of Southern California

Los Angeles, CA 90089

rothemun@pollux.usc.edu

Received for publication November 9, 1997; accepted as revised October 18, 1998. 